\title{
Case Study To Show The Effectiveness Of Dry Needling In The Treatment Of Insertional Supraspinatus Calcific Tendinopathy-Using Radiography As A Diagnostic Technique
}

\section{Diana Pinto*, Prachi Kulkarni, and Rokade}

Physiotherapy, Nutrition and Pain Management Institute, Mumbai, India

\begin{abstract}
Dry Needling is gaining wide popularity as a technique to relieve musculoskeletal pain. It has rarely been used to treat Supraspinatus Calcific tendinopathy. Most treatment techniques focus around Subacromial Corticosteroid needle decompression to improve the outcomes of Supraspinatus Calcification. Our study observed the Elastographic changes along with the Radiographic changes in the Calcification before and after the Dry Needling sessions.
\end{abstract}

Corresponding author: Diana Pinto, Physiotherapy, Nutrition and Pain Management Institute, Mumbai, India. E-mail: diana.j.pinto@gmail.com

Received: April 30, 2020 ; Accepted: May 07, 2020 ; Published: May 10, 2020

Keywords: Shoulder pain, Dry Needling, Supraspinatus, Calcification, AP Radiograph

\section{Introduction}

Urbanization and the diversity of occupation has led to an increase in musculoskeletal injuries on account of lifting heavy loads, repetitive joint movements in abnormal positions, and vibrations from heavy machinery. Such injuries influence the level of symptoms and disability in patients. Shoulder injuries are the third most reported musculoskeletal complaint after low back pain and knee pain. Shoulder pain is reported to be the third most common cause of musculoskeletal complaints amongst populations [1-3]. A review of Literature states that the conventional line of Physiotherapy treatment for shoulder complaints includes techniques like man- ual therapy, exercise therapy, electrotherapy - although each reported condition had a specific technique of treatment or required an adjunct technique for its complete recovery. The Dry Needling technique is fast gaining prominence as a means towards symptomatic relief and complete recovery of the diagnosed Musculoskeletal issues. Several studies have focused on the use of Dry Needling to bring about symptomatic relief in various Musculoskeletal conditions.

Our Case report uses the Dry Needling technique to not only provide symptomatic relief to the patient, but also bring about an observable improvement in the appearance of Cal- 
Citation: Diana Pinto, Prachi Kulkarni, Rokade (2020) Case Study To Show The Effectiveness Of Dry Needling In The Treatment Of Insertional Supraspinatus Calcific Tendinopathy-Using Radiography As A Diagnostic Technique. Journal of Physical Medicine Rehabilitation Studies \& Reports. SRC/JPMRS/106. DOI: doi.org/10.47363/JPMRS/2020(2)105

cium deposits in a patient with Calcific Supraspinatus Tendinopathy.

\section{Methods and Materials}

Calcific Tendonitis is common in the Supraspinatus muscle (sometimes the Shoulder Rotator cuff muscles), the Achilles and the Patellar tendons. Calcium deposits in the substance of the Supraspinatus tendon worsen the clinical manifestations of Supraspinatus Tendinopathy, thus contributing to an increase in rupture rate and slower recovery times. The Etiopathogenesis of Calcific Tendinopathy is debatable, but could be attributed to an active cell mediated process brought on by an inflamed tendon to locally compensate for the original decreased stiffness [4]. Chiou et al, found a statistically significant correlation between the morphology of the Calcified deposits and the clinical symptoms reported by the patient [5]. They classified these calcific deposits into four shapes according to Ultrasonographic findings: a) Arc shape; b) Fragmented/ Punctuated shape; c) Nodular shape; and 4) Cystic shape. Gärtner on the other hand radiologically classified Calcifying Tendinitis as follows [6].

\begin{tabular}{|l|l|}
\hline Type I & Homogeneous structure, sharp outline \\
\hline Type II & $\begin{array}{l}\text { Inhomogeneous structure, sharp outline, } \\
\text { or homogeneous structure, no defined } \\
\text { outline }\end{array}$ \\
\hline Type III & $\begin{array}{l}\text { Inhomogeneous structure, no defined } \\
\text { outline }\end{array}$ \\
\hline
\end{tabular}

Table 1: Radiological classification of calcifying tendinitis (Gärtner).

A 42 year old Male patient manifested with chronic activity related pain at his Right shoulder since 8 months, localized tenderness at the tip of his Shoulder and a decreased range of motion in Active Shoulder abduction and resisted medial rotation. Patient had tried Ultrasound treatment and analgesics during those 8 months and found no symptomatic relief. The patient was a Type III on Gärtner's Radiological Classification scale and had Fragmented Calcific deposition at the bone tendon junction [Figure 1].

Anteroposterior Radiographic view of the Right Shoulder was taken at the patient's first visit (Parameters: $25 \mathrm{mAs}$, $55 \mathrm{kVp}, 200 \mathrm{~mA}$ ). An Ultrasonogram of his Right shoulder was also done, with focus on the Calcifications. Patient was in sitting position with his Right forearm behind his back.
The Ultrasound study described the Supraspinatus tendon as mildly bulky along with multiple calcific foci around the Supraspinatus tendon insertion. There was no evidence of Tendon tear/ Bursal distension.

\section{Procedure}

After proper explanation of the Dry Needling procedure to the patient, one time use disposable needles were used to the following muscles with the patient in Left side lying position, right arm on top but supported behind his body with the help of pillows:

- Deltoid (Anterior, Posterior and Middle) muscle

- Supraspinatus muscle and its insertion at Greater Trochanter

Immediately after treatment, patient was advised to do gentle free range movements in order to decrease the discomfort from the needling. Patient took Dry Needling therapy to the above mentioned structures, once a week for 5 months. His symptoms of pain during movement decreased significantly in the first 2 weeks of treatment. Patient reported complete improvement in pain during active shoulder abduction after 4 weeks of treatment. There was no pain in resisted medial rotation of shoulder at the end of 5 weeks. A radiograph at the end of 8 weeks revealed decreased appearance of Calcifications at the Right shoulder. Patient requested to continue with treatment until all Calcific deposits cleared out on Radiograph.

Although CT scans of the rotator cuff shows a tendency toward spontaneous resorption of the deposits and symptoms often resolve spontaneously without any form of treatment, some authors describe persistent pain at long time follow-up and persistent reduction of ROM in patients with Calcific tendonitis $[7,8]$. Patient came in once a month for Dry Needling sessions after the end of 8 weeks of initial treatment. At the end of 20 weeks, a Radiograph revealed improvement in Calcifications around his Right shoulder with complete range of pain free movement at the shoulder in active and resisted movements [Figure 2]. Patient was discharged from care at the end of 11 treatment sessions. 


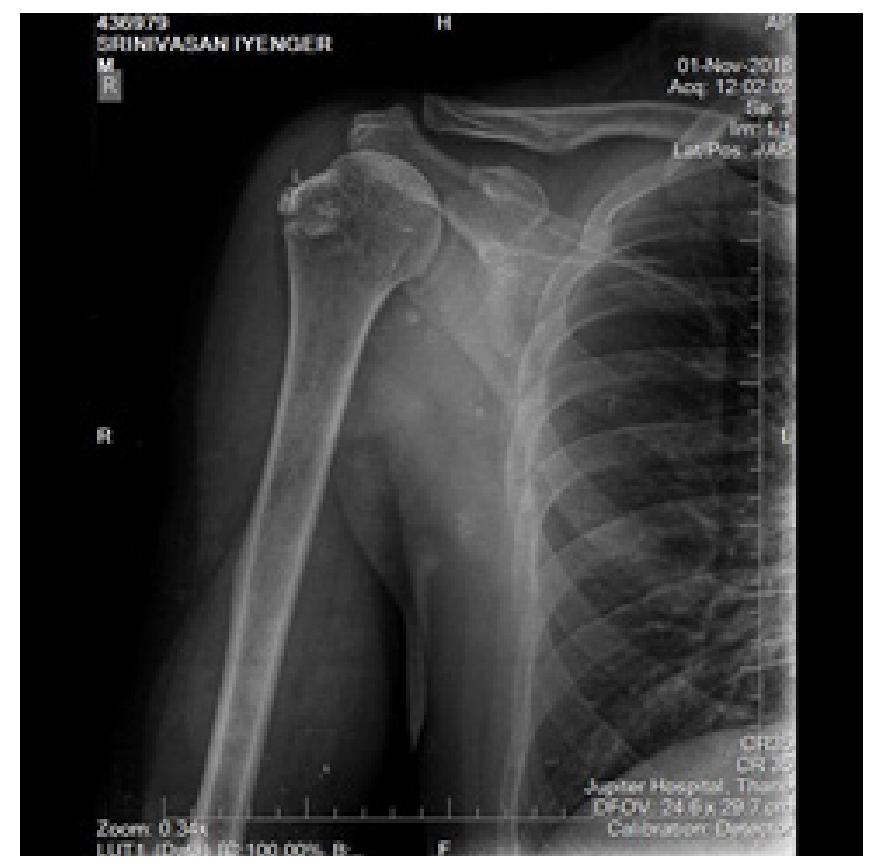

Figure 1: AP view Radiograph taken prior to initiation of Dry Needling therapy.

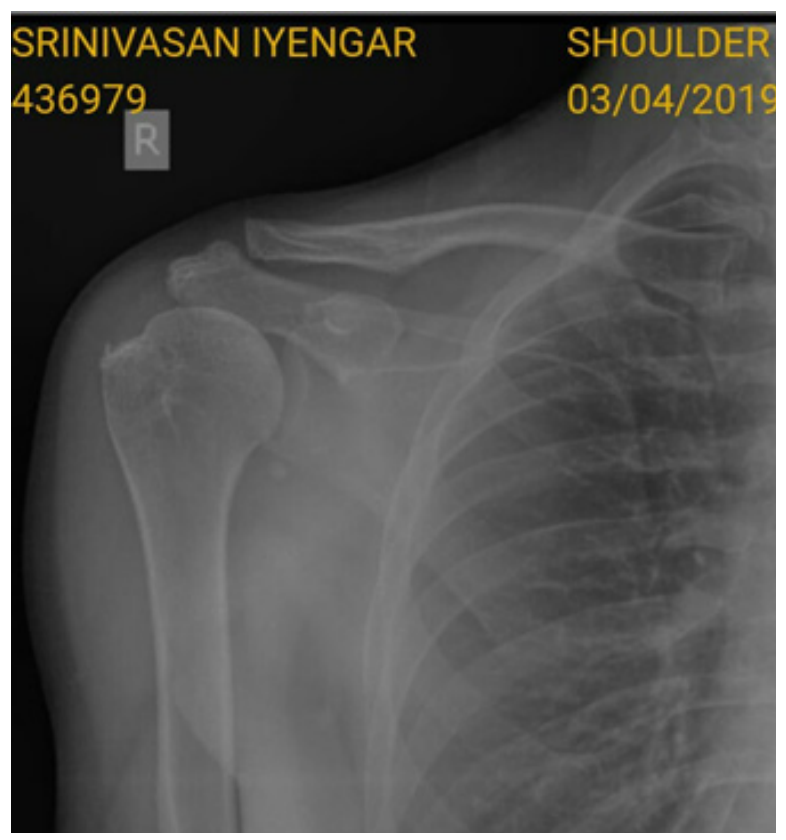

Figure 2: AP view Radiograph taken after 11 sessions of Dry Needling therapy to Right Shoulder.

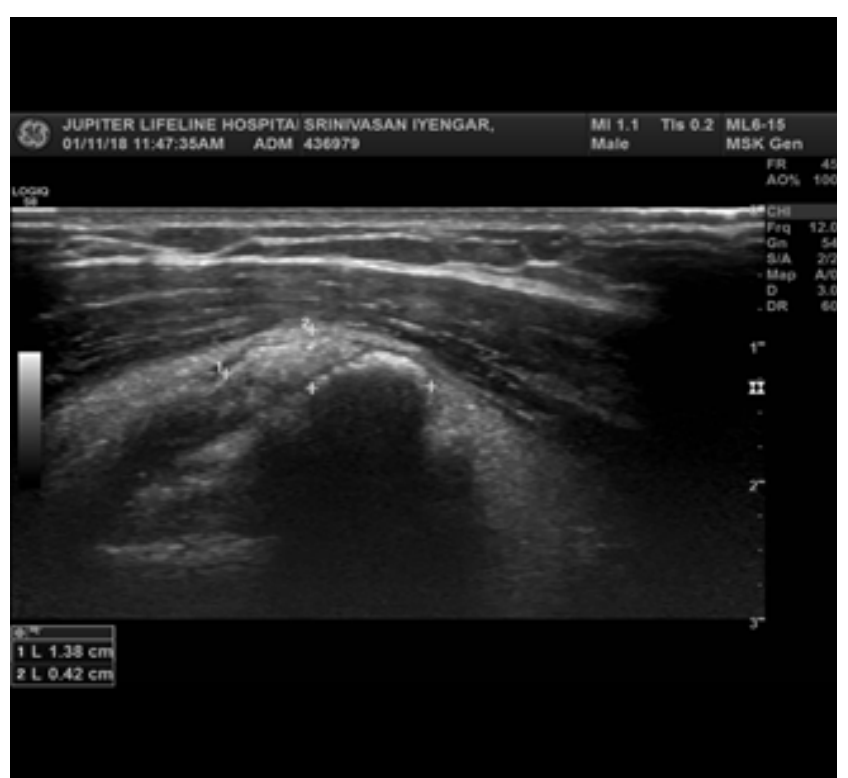

Figure 3: Ultrasonography of Right Calcific Tendonitis of Right shoulder prior to Dry Needling.

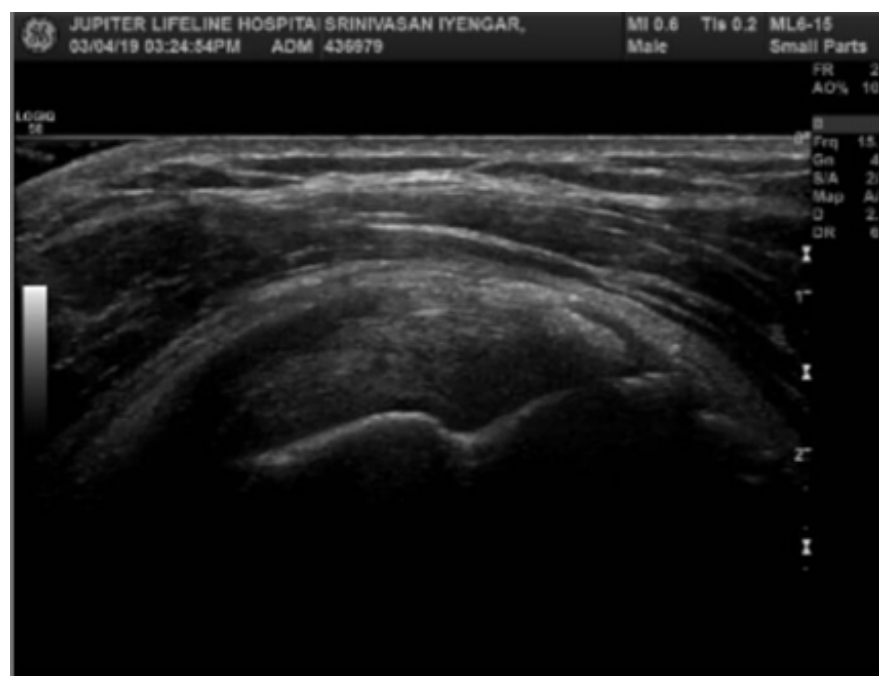

Figure 4: Ultrasonography of Right Calcific Tendonitis of Right shoulder after 11 sessions of Dry Needling.

\section{Results}

Patient reported symptomatic relief of pain in both active and resisted movements of shoulder within five sessions, over five weeks of Dry Needling treatment. Radiograph of the symptomatic AP Shoulder showed significant decrease in the appearance of Calcific deposits after 11 sessions of Dry Needling treatment over 5 months. Follow through with the patient one year later in March 2020, over phone call (due to Lockdown and Social distancing protocol on account of 
Citation: Diana Pinto, Prachi Kulkarni, Rokade (2020) Case Study To Show The Effectiveness Of Dry Needling In The Treatment Of Insertional Supraspinatus Calcific Tendinopathy-Using Radiography As A Diagnostic Technique. Journal of Physical Medicine Rehabilitation Studies \& Reports. SRC/JPMRS/106. DOI: doi.org/10.47363/JPMRS/2020(2)105

COVID-19) revealed that patient had no return of pain after the last Dry Needling session in April 2019. Patient did not experience pain during active abduction movement of his Right Shoulder.

Simultaneous Ultrasonogram images have been provided for before needling and after Dry needling sessions. Follow up Ultrasonography scan of Right Supraspinatus tendon showed a mildly bulky tendon with very one minor calcific focus around the insertion of the Supraspinatus tendon. Elastography which was done alongside the Ultrasonogram (but not reported in this Case report) showed a decrease in Elastography ratio of Deltoid muscle/Supraspinatus tendon of 0.3 at the beginning of treatment to 0.2 after 11 sessions of Dry Needling treatment.

\section{Conclusion}

To our knowledge there was only one existing paper by Roy Settergren [9] that addressed the changes in Echogenecity of Supraspinatus tendon, achieved through Dry Needling procedures as was observed on Diagnostic Ultrasonography imaging.

The drawbacks of our study were:

1. Our study focused on Dry Needling techniques without using Ultrasound guided imaging during Acupuncture needle insertion into pathologic tissues. An Ultrasound guided Needling technique could perhaps have helped us improve the mild bulkiness of the Supraspinatus tendon.

2. It was found imperative by us, to include the Dry Needling of other shoulder muscles that contribute to the improvement of biomechanics in a pathologic shoulder - for any future Calcific tendonitis case studies. Dry Needling of these muscles could possibly hasten the healing of an underlying tendinopathy and associated calcification.

Introduction of a Dry Needle into pathologic tissue is known to induce a humoral healing response. However further research is needed into understanding the mechanism behind the Resorption of a long standing Fragmented Calcific (Insertional) Supraspinatus Tendinopathy through Dry Needling. As for the case report presented in this paper, Dry Needling was shown to be beneficial for this patient as was evident by the Radiologic changes pre and post procedure, and no return of symptoms post one year of discharging from care. This author suggests that a proper Ultrasound guided Dry Needling procedure be considered for future studies to treat Calcific tendinopathies.

\section{References}

1. Michelle Urwin, Deborah Symmons et al (1998) Estimating the burden of musculoskeletal disorders in the community: the comparative prevalence of symptoms at different anatomical sites, and the relation to social deprivation 57:649-655.

2. Sherise Epstein, Emily H. Sparer, Bao N. Tran (2018) Prevalence of Work-Related Musculoskeletal Disorders Among Surgeons and Interventionalists: A Systematic Review and Meta-analysis 153(2).

3. Waleed Umer, Maxwell F. Antwi-Afari, Heng Li, Grace P. Y. Szeto, Arnold Y. L. Wong (2018) The prevalence of musculoskeletal symptoms in the construction industry: a systematic review and meta-analysis 125-144.

4. Francesco Oliva, Alessio Giai Via, Nicola Maffulli (2012) Physiopathology of intratendinous calcific deposition 10:95

5. Chiou HJ, Hung SC, in SY, Wei YS, Li MJ (2010) Correlations among mineral components, progressive calcification process and clinical symptoms of calcific tendonitis 49:548-555.

6. Gärtner J (1993) Tendinosis calcarea: results of treatment with needling. Z Orthop Ihre Grenzgeb 131:461-9.

7. Chan R, Kim DH, Millett PJ, Weissman BN (2004) Calcifying tendinitis of the rotator cuff with cortical bone erosion 33:596-599.

8. Flemming DJ, Murphey MD, Shekitka KM, Temple HT, Jelinek JJ et al (2003) Osseous involvement in calcific tendinitis: a retrospective review of 50 cases 181:965-972.

9. Roy Settergren (2013) Treatment of supraspinatus tendinopathy with ultrasound guided dry needling 26-29.

Copyright: @2020 Diana Pinto. This is an open-access article distributed under the terms of the Creative Commons Attribution License, which permits unrestricted use, distribution, and reproduction in any medium, provided the original author and source are credited. 